\title{
Treatment Difficulties in High Risk Pulmonary Embolism. A Case Report
}

\author{
Balázs Bajka' ${ }^{1}$ Edvin Benedek ${ }^{2}$, Alexandra Stănescu² ${ }^{2}$ Emese Rapolti ${ }^{1}$, Monica Chițu${ }^{1}$, \\ István Kovács ${ }^{1}$ \\ ${ }^{1}$ Clinic of Cardiology, University of Medicine and Pharmacy of Tirgu Mures, Romania \\ ${ }^{2}$ Center of Advanced Research in Multimodality Cardiac Imaging, Cardio Med Medical Center, Tîrgu Mureș, Romania
}

\begin{abstract}
Pulmonary embolism (PE) remains a common and potentially life-threatening cardiovascular emergency. Systemic thrombolysis with intravenous infusion of a thrombolytic agent is generally recommended for treatment of high risk PE. However, this method has known limitations in the presence of high bleeding risk. Catheter-directed thrombolysis has the potential to achieve the same benefits as systemic thrombolysis, with a lower risk of haemorrhage. The case presented is of a 67-year-old male patient with a high risk of pulmonary embolism and contraindications for systemic thrombolysis, in whom the presence of severe comorbidities presented an increased risk of surgical embolectomy, who was successfully treated by catheter-directed thrombolysis.
\end{abstract}

Keywords: pulmonary embolism, thrombolysis, embolectomy

\section{ARTICLE HISTORY}

Received: 15 October, 2015

Accepted: 17 January, 2016

\section{CORRESPONDENCE}

Edvin Benedek

76, 22 decembrie 1989 St

540124 Tîrgu Mureș, Romania

Tel: +40-265-217333

Email: edvinke87@gmail.com

\section{INTRODUCTION}

Acute pulmonary embolism (PE) is a life-threatening condition which has an estimated global mortality rate of $15 \%$ at 3 months. ${ }^{1}$ High risk pulmonary embolism is defined as an acute PE associated with persistent systemic arterial hypotension, a systolic arterial pressure $<90 \mathrm{mmHg}$, the requirement of an inotropic agent, cardiogenic shock, or a condition progressing to the need for cardiopulmonary resuscitation. In severe cases of high risk $\mathrm{PE}$, the in-hospital mortality rate may exceed $50 \%{ }^{2}$

Systemic thrombolysis with intravenous infusion of a thrombolytic agent is generally recommended for treatment of high risk PE. However a meta-analysis of ran- domized clinical trials of thrombolytic treatment versus heparin-only treatment, demonstrated no significant reduction in death rates due to an increase in the number of haemorrhagic events. ${ }^{3-5}$ In addition, systemic thrombolysis has absolute and relative contraindications. Absolute contraindications include prior intracranial haemorrhage, known intracranial cerebrovascular disease, known malignant intracranial neoplasm, ischemic stroke within the previous three months, aortic dissection, active bleeding or bleeding diathesis, or recent surgery encroaching on the spinal canal or brain. Relative contraindications are age $>75$ years, current use of anticoagulation therapy, pregnancy, puncture of a non-compressible vessel, traumatic or prolonged cardiopulmonary resuscitation $(>10$ 
TABLE 1. Laboratory findings in the emergency room

\begin{tabular}{lcc}
\hline Laboratory findings & Value & Reference range \\
\hline D dimer & $>5 \mu \mathrm{gg} / \mathrm{ml}$ & $<0.5 \mu \mathrm{gg} / \mathrm{ml}$ \\
CTnI & $0.846 \mathrm{ng} / \mathrm{ml}$ & $<0.02 \mathrm{ng} / \mathrm{ml}$ \\
NTproBNP & $974 \mathrm{pg} / \mathrm{ml}$ & $<400 \mathrm{pg} / \mathrm{ml}$ \\
Hemoglobin & $11.6 \mathrm{~g} \%$ & $14-17 \mathrm{~g} \%$ \\
Hematocrit & $36.1 \%$ & $40-48 \%$ \\
ASAT & $66 \mathrm{U} / \mathrm{I}$ & $<40 \mathrm{U} / \mathrm{I}$ \\
ALAT & $42 \mathrm{U} / \mathrm{I}$ & $<38 \mathrm{U} / \mathrm{I}$ \\
\hline
\end{tabular}

minutes), internal bleeding within the previous two to four weeks, history of chronic, severe, and poorly controlled hypertension, severe uncontrolled hypertension on presentation, dementia, remote ischemic stroke of more than three months duration or major surgery within three weeks. ${ }^{3}$

Whenever absolute contraindications for thrombolysis are present, surgical embolectomy could represent an alternative option to reperfusion treatment. With a multidisciplinary approach and individualized indications for embolectomy, perioperative mortality rate is less than $6 \% .{ }^{6}$ However, this is a major intervention, requiring cardiopulmonary bypass for extraction of the obstructing pulmonary emboli.
In cases with absolute contraindications to systemic thrombolysis, an interventional approach could be considered as an alternative to reperfusion instead of surgical embolectomy. An interventional approach may include thrombus fragmentation with either pigtail or balloon catheters, rheolytic thrombectomy with hydrodynamic catheter devices, suction thrombectomy with aspiration catheters or rotational thrombectomy. Catheter-directed thrombolysis or pharmacomechanical thrombolysis seem to be the preferred approaches for patients without absolute contraindications for thrombolytic therapy. ${ }^{7}$

The case presented is of a 67-year-old male patient having a high risk of pulmonary embolism and contraindications for systemic thrombolysis, in whom the presence of severe comorbidities led to an increased risk of surgical embolectomy, who was successfully treated by catheter-directed thrombolysis.

\section{CASE PRESENTATION}

A 67-year-old man presented to the emergency department with severe shortness of breath which had commenced abruptly the day before admission, followed by collapse without loss of consciousness. Associated symptoms were chest pain and diaphoresis.

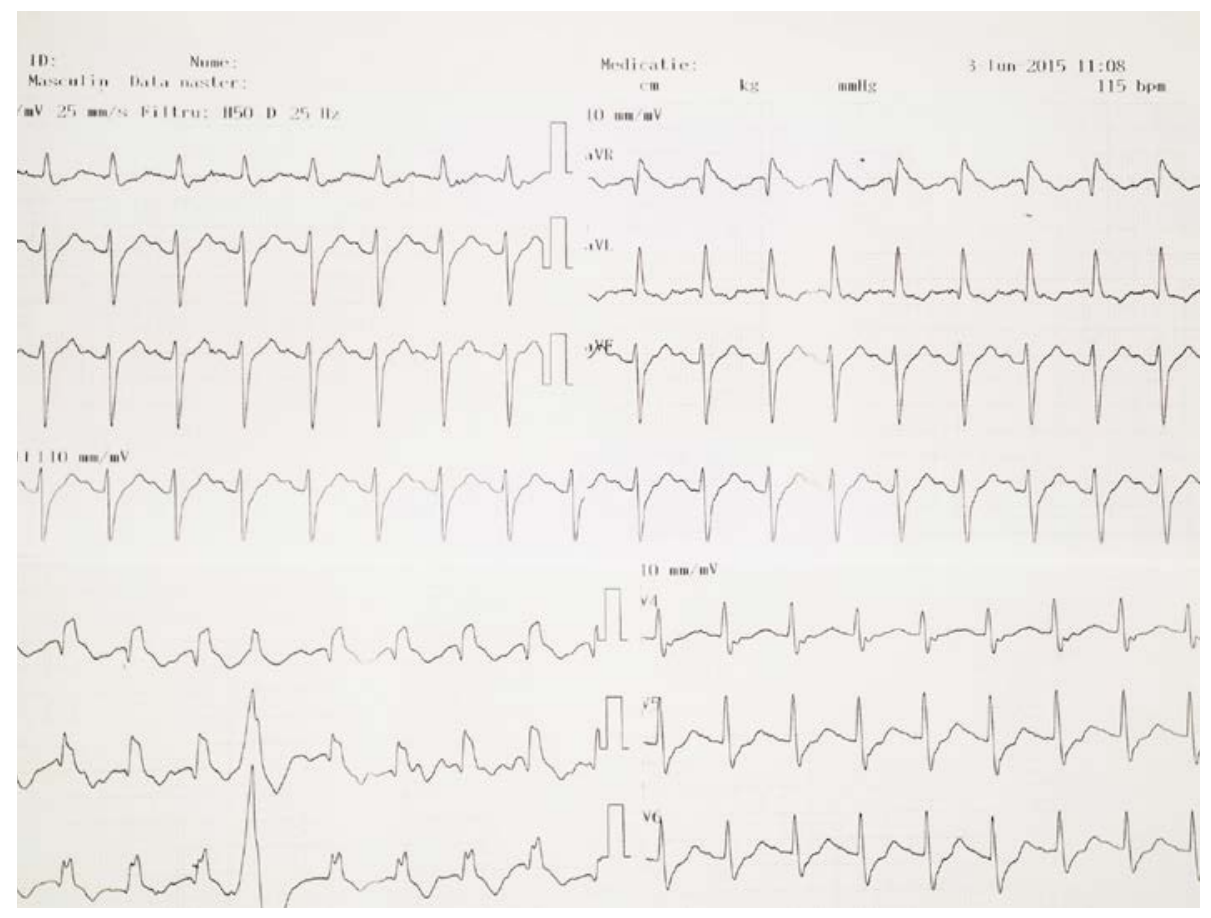

FIGURE 1. Initial electrocardiogram in the emergency room. Sinus tachycardia at rate of 115 beats per minute, bifascicular block - right bundle branch block (QRS duration of $140 \mathrm{msec}$ ) and LAH, left axis deviation, diffuse ST-segment depression, and T-wave inversion in leads $\mathrm{V}_{1}-\mathrm{V}_{3}$. 


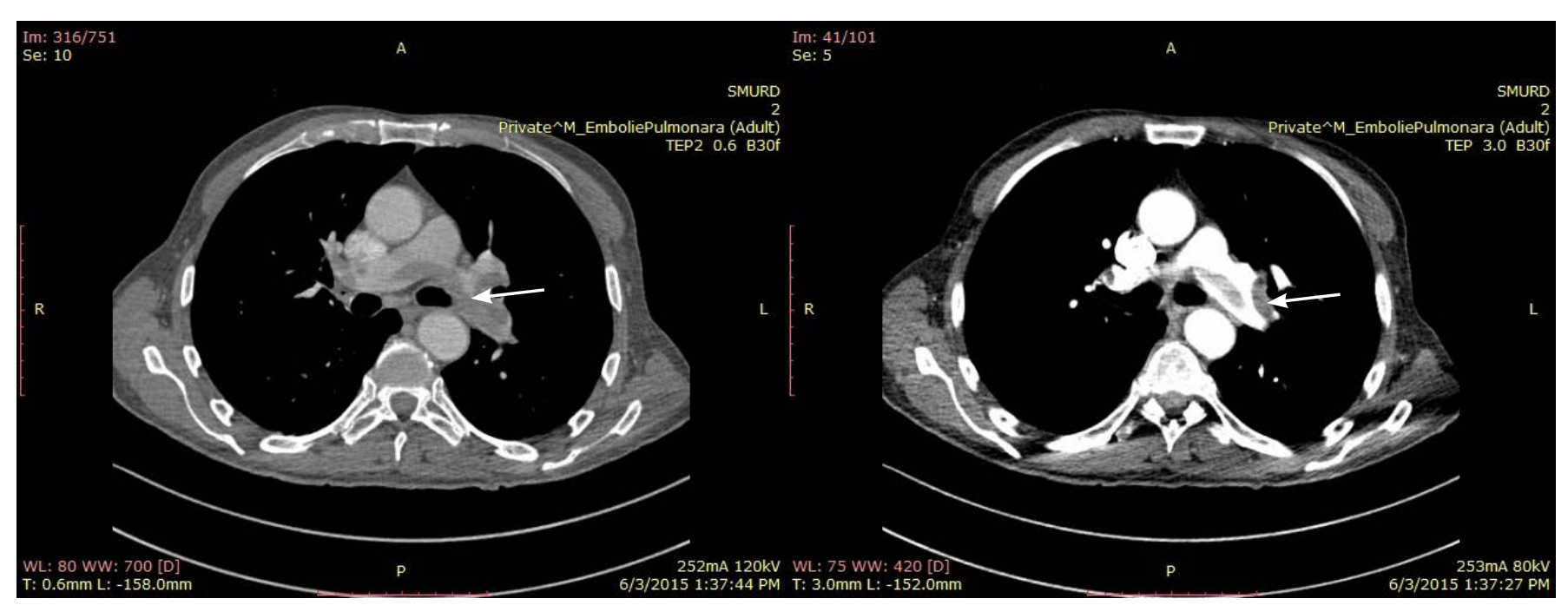

FIGURE 2. Axial computed tomographic pulmonary angiogram showing the large filling defect at the bifurcation of the main pulmonary artery (A) as well as multiple subsegmental clots (B).

The informed consent was obtained from the patient for publication of this case report and any accompanying images. The publication of this case was accepted by the Ethics Committee of the hospital.

The relevant medical history included a right-sided hemiparesis occuring two months before, a recurent ischemic stroke complicated by an intraparenchimal haematoma, which had required neurosurgical intervention, and stage 3 essential hypertension for which he was receiving medication. His social history revealed an alcohol consumption of approximately one bottle of wine daily for twenty years. He was a non-smoker. The patient was on antihypertension therapy, quinapril $20 \mathrm{mg}$ and metoprolol $25 \mathrm{mg}$, both twice daily, indapamid $1.5 \mathrm{mg}$ once a day. He had no known drug allergies.

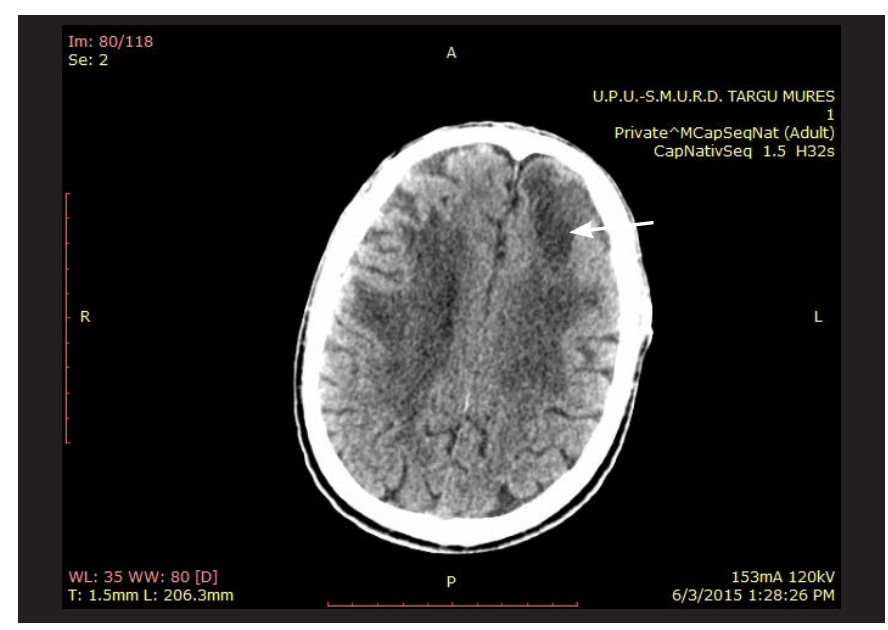

FIGURE 3. Native CT of the head: without intracranial bleeding, ischemic lesion in the frontal lobe with dimension of $28 \mathrm{~mm}$
His vital signs showed a systolic blood pressure of 90/60 mmHg, a heart rate of 134 beats per minute, a respiratory rate of 37 per minute with an oxygen saturation of $80 \%$ when breathing air and $91 \%$ when using a $100 \%$ non-rebreather facemask. He was pale, diaphoretic, and his jugular veins were distended to the angle of the jaw. Cardiac examination showed extreme tachycardia with fixed widening of the second heart sound, the presence of a third heart sound and a right ventricular heave. The extremities were cold and cyanotic, with weak peripheral pulses. An electrocardiogram showed sinus tachycardia at a rate of 115 beats per minute, bifascicular block - right bundle branch block with a QRS duration of $140 \mathrm{msec}$, and left anterior hemiblock with left axis deviation, and diffuse ST-segment depression and T-wave inversion in leads V1-V3 (Figure 1).

The arterial blood gas analysis showed a $\mathrm{pH}$ of 7.54, $\mathrm{pCO}_{2}$ of $28.3 \mathrm{mmHg}, \mathrm{pO}_{2}$ of $40.2 \mathrm{mmHg}$ and $77.8 \%$ oxygen saturation.

Bedside echocardiography demonstrated severe right ventricular (RV) dilation with signs of RV volume and pressure overload, severe hypokinesis of the RV free wall and the ventricular septum, and good RV apical contraction with a positive McConnel sign. The left ventricle (LV) systolic function was well-preserved with no evidence of abnormal wall movement. The pulmonary artery pressure was estimated to be $45 \mathrm{mmHg}$, assuming a right atrial pressure of $10 \mathrm{mmHg}$. The laboratory findings showed a D-dimer value greater than $5 \mathrm{\mu g} / \mathrm{ml}$, elevated cTnI ( 0.846 $\mathrm{ng} / \mathrm{ml}$, reference range $<0.02 \mathrm{ng} / \mathrm{ml}$ ) and NTproBNP (974 $\mathrm{pg} / \mathrm{ml}$, reference range $<400 \mathrm{pg} / \mathrm{ml}$ ), mild anemia (Htc 11.6 g\%, Htc $36.1 \%$ ) with slightly elevated liver enzymes 


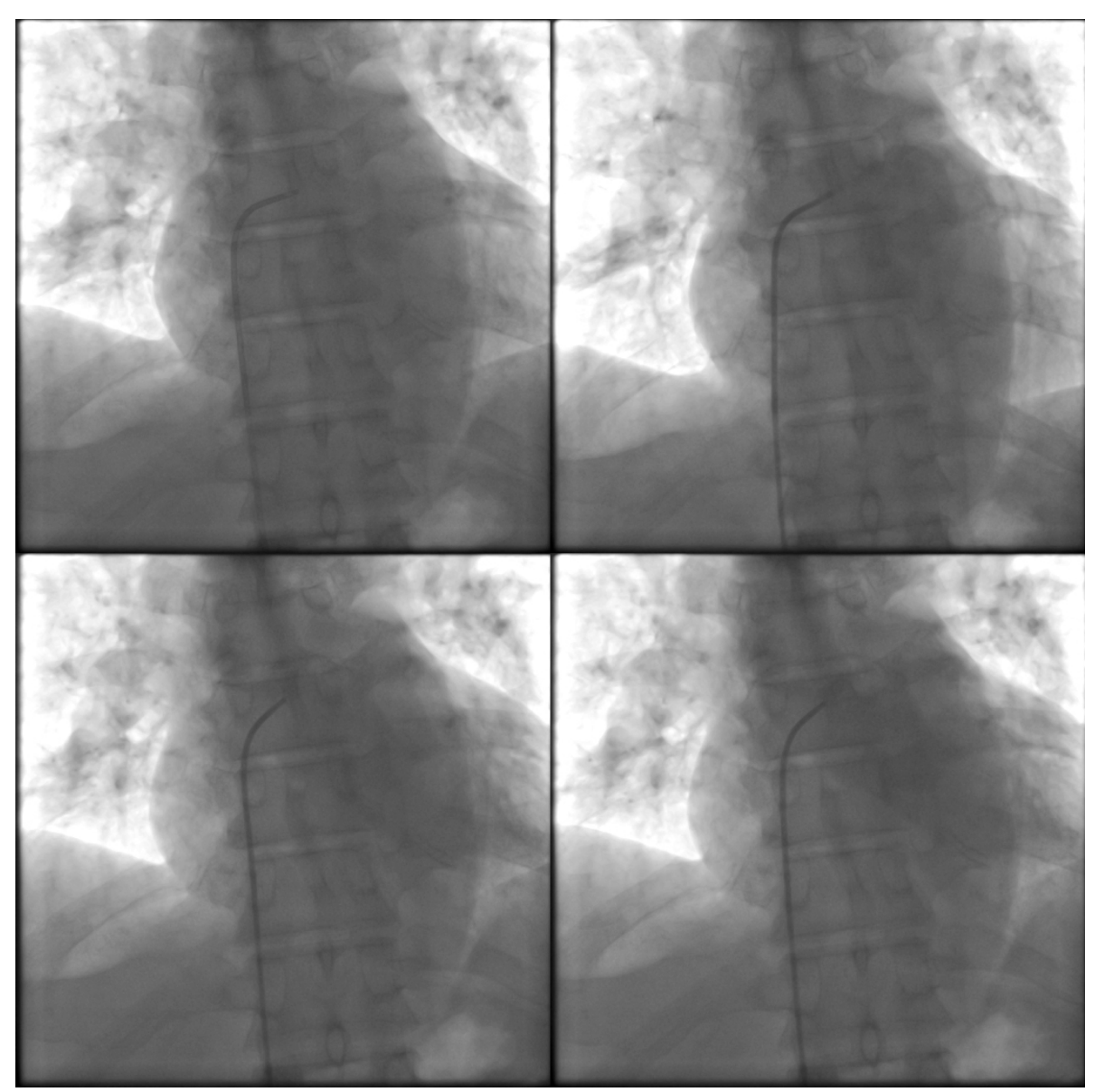

FIGURE 4. The accidentally found ASD. The JL4 catheter is inserted into the left atrium without resistance, the contrast media is injected to the left heart and in the aorta.

(ASAT 66U/L, referece range < 40 U/L, ALAT 42 U/L, reference range $<38 \mathrm{U} / \mathrm{L}$ ) (Table 1 ).

The patient was immediately given a saline infusion and a loading dose of 5000 IU intravenous heparin for a suspected pulmonary embolism. The patient's blood pressure continued to decrease, therefore a dopamine infusion was initiated at a rate of 7.5 micrograms $/ \mathrm{kg} /$ minute.

The computed tomogram (CT) showed a large filling defect at the bifurcation of the main pulmonary artery with a diameter of $11 \mathrm{~mm}$, and two filling defects at the origin of the right pulmonary artery and left pulmonary artery, havixng dimensions of $26 \times 22 \mathrm{~mm}$ and $17 \times 14$ $\mathrm{mm}$ respectively, as well as multiple sub-segmental clots (Figures 2A and B). The native head CT was negative for intracranial bleeding (Figure 3). Considering the haemodynamic compromise resulting from the massive pulmonary embolism, and the high risk of intracranial bleeding due to the neurosurgical medical history, the local heart team decided to take an interventional approach for the treatment of this complicated case.
Pulmonary angiogram was performed using a Judkins Left 4.0 guiding coronary catheter introduced via the femoral vein. On reaching the right atrium, the catheter passed into the left atrium without any resistance, through a previously unidentified atrial septal defect. After being injected into the right atrium, contrast media passed from the right atrium to the left heart and the aorta (Figure 4). After insertion of the catheter into the main pulmonary artery, the pulmonary angiogram confirmed the presence of massive emboli in the pulmonary circulation (Figure 5).

Knowing the fact that the administration of rtPA is associated with a higher incidence of intracranial bleeding, streptokinase (SK) was given in a half dose of 125.000 IU over thirty minutes, followed by $50.000 \mathrm{IU} / \mathrm{h}$ for twelve hours. Following the infusion of thrombolytic therapy, the patient's blood pressure increased to $115 / 80 \mathrm{mmHg}$, at which point the administration of dopamine was discontinued. The tachycardia decreased to $85 / \mathrm{min}$, shortness of breath diminished, and oxygen saturation was $>94 \%$ on breathing air. Post-thrombolysis ECG showed sinus 


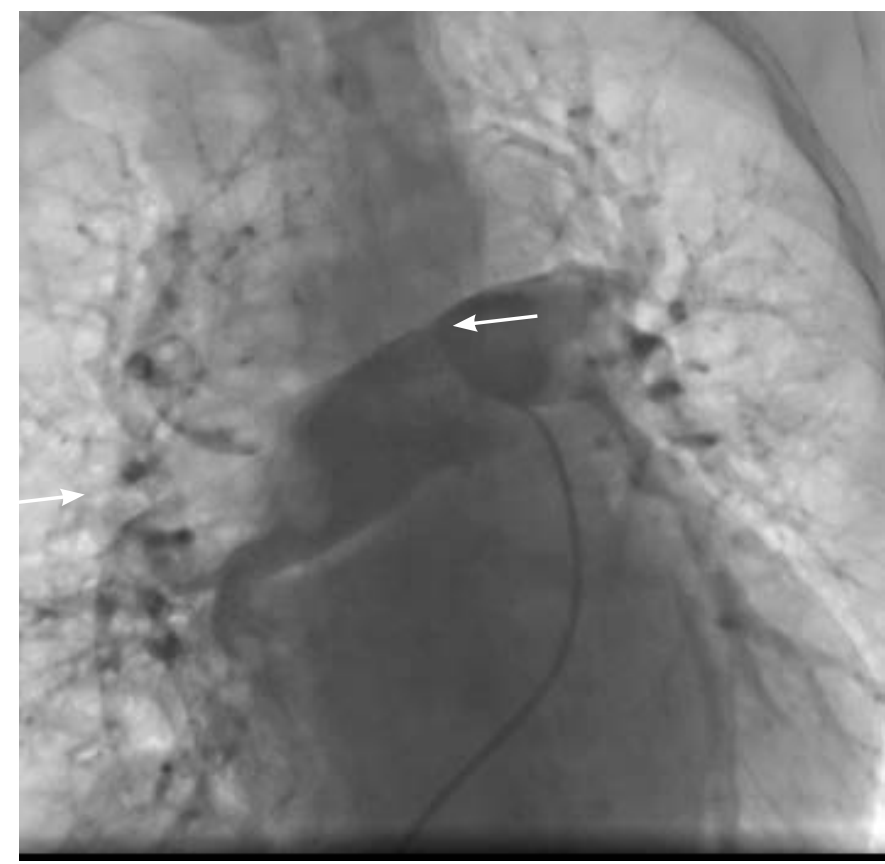

FIGURE 5. The pulmonary angiogram showed large filling defect in the bifurcation of the right and left pulmonary arteria

rhythm with a frequency of $85 /$ min, right bundle branch block and left atrial hypertrophy (Figure 6).

\section{DISCUSSIONS}

Heparin is considered the cornerstone of management of $\mathrm{PE}$, accelerating the action of antithrombin III, preventing additional thrombus formation and allowing endogenous fibrinolysis to dissolve a significant part of a clot. ${ }^{8}$

Fibrinolysis can be life-saving in cases of massive pulmonary embolism, in the presence of haemodynamic instability or in cases of cardiogenic shock, due to thrombolytic agents causing the lysis of a clot. Several studies show clear benefits of thrombolysis in patients with high risk PE, leading to a significant improvement in haemodynamic status, oxygen saturation, and a lower incidence of early PE recurrence. 4,5

Administration of fibrinolytic agents increases the risk of major bleeding, including intracranial haemorrhage. Analysis of data derived from clinical trials using various thrombolytic agents indicates an incidence of intracranial bleeding between $1.9 \%$ and $2.2 \% .{ }^{9,10}$ Increasing age and the presence of comorbidities have been associated with a higher risk of bleeding complications following thrombolysis, indicating the need of a safer management of patients with increased risk of intracranial haemorhage or other life-threatening bleeding disorders. ${ }^{11}$

According to a study that included 121 patients with moderate PE, use of a reduced-dose rtPA regimen seems to be a safer strategy in the treatment of intermediate-tohigh risk PE. ${ }^{12,13}$

An alternative approach for patients with high risk PE with contraindications for a full dose, is systemic thrombolytic therapy consisting of local, catheter-delivered small doses of a thrombolytic agent. A metaanalysis of 35 non-randomized interventional approach studies,

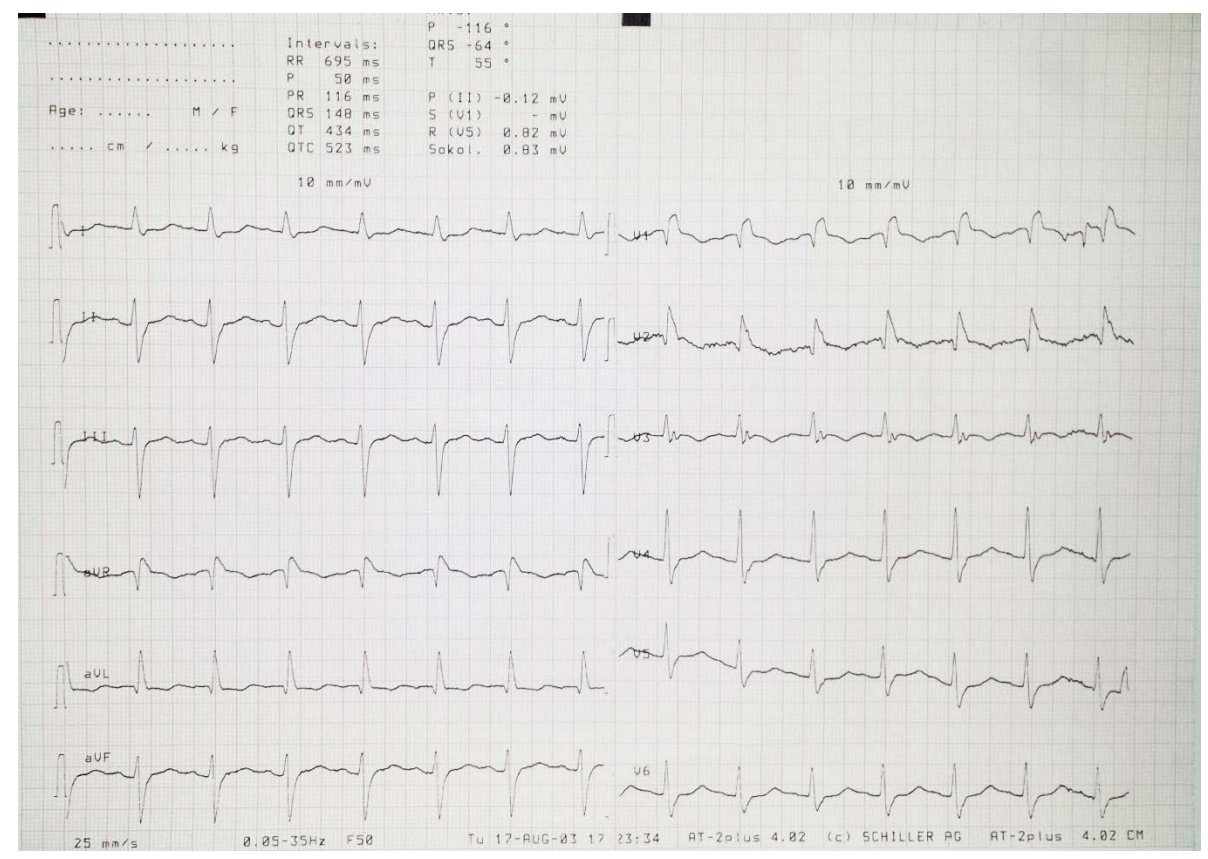

FIGURE 6. ECG after selective trombolysis. Sinus rhythm $85 / \mathrm{min}, \mathrm{RBBB}, \mathrm{LAH}$ 
reporting on a total of 594 patients with high risk $\mathrm{PE}$, showed an $87 \%$ clinical success with stabilization of haemodynamic parameters, hypoxia reduction and survival up to the point of discharge. ${ }^{7}$ The effect of the low-dose catheter-directed thrombolysis on recovery of right ventricular function appears comparable to the one obtained using standard-dose systemic thrombolysis, with a lower risk of intracranial or any kind of life-threatening bleeding. ${ }^{14,15}$

The current case is remarkable due to the presence of a massive PE in association with contraindications for systemic thrombolysis and severe comorbidities, which led to an inacceptable high risk for surgical embolectomy. In cases when alteplase administraion is contraindicated or there is insufficient time for full-dose administration, catheter-directed thrombolysis may be the only practicable treatment option. In experienced centers, the use of modern catheter-directed thrombolitic therapy has proven to be a life-saving treatment in patients dying from acute massive PE. ${ }^{7}$

\section{CONCLUSIONS}

Pulmonary embolism is a potentially life-threatening cardiovascular emergency associated with significant morbidity and mortality both in the early and late stages.

In patients presenting with high risk $\mathrm{PE}$ and associated severe comorbidities which lead to an increased risk of major bleeding, catheter-directed thrombolysis represents a feasible alternative strategy to surgical embolectomy. Patients treated with selective thrombolytic therapy show rapid improvement of right ventricular function and pulmonary perfusion which may lead to a lowering of early recurrent $\mathrm{PE}$ and decreased risk of developing late sequelae such as chronic pulmonary hypertension.

\section{CONFLICT OF INTEREST}

Nothing to declare.

\section{REFERENCES}

1. Goldhaber SZ. Pulmonary Embolism. N Engl J Med. 1998;339:93-104.

2. Goldhaber SZ. Thrombolysis for pulmonary embolism. N Engl J Med. 2002;347:1131-1132. doi:10.1016/0033-0620(91)90009-B.
3. Fiumara K, Kucher N, Fanikos J, Goldhaber SZ. Predictors of major hemorrhage following fibrinolysis for acute pulmonary embolism. Am J Cardiol. 2006;97:127-129. doi: http://dx.doi. org/10.1016/j.amjcard.2005.07.117.

4. Goldhaber SZ, Haire WD, Feldstein $\mathrm{ML}$, et al. Alteplase versus heparin in acute pulmonary embolism: randomized trial assessing right ventricular function and pulmonary perfusion. Lancet. 1993;341:507-511. doi:10.1016/01406736(93)90274-K.

5. Konstantinides S, Tiede N, Kasper W, et al. Comparison of alteplase versus heparin for resolution of major pulmonary embolism. Am J Cardiol. 1998;82(8):966-970. doi: http:// dx.doi.org/10.1016/S0002-9149(98)00513-X.

6. Fukuda I, Taniguchi S, Suzuki Y, et al. Improved outcome of surgical pulmonary embolectomy by aggressive intervention for critically ill patients. Ann Thorac Surg. 2011;91(3):728-732. doi: 10.1016/j.athoracsur.2010.10.086.

7. Kuo WT, Gould MK, Hofmann LV, et al. Catheter-directed therapy for the treatment of massive pulmonary embolism: systematic review and meta-analysis of modern techniques. J Vasc Interv Radiol. 2009;20(11):1431-1440. doi: 10.1016/j. jvir.2009.08.002.

8. Barritt DW, Jordan SC. Anticoagulant drugs in the treatment of pulmonary embolism: a controlled trial. Lancet. 1960;1(7138):1309-1312.

9. Kanter DS, Mikkola KM, Goldhaber SZ, et al. Thrombolytic therapy for pulmonary embolism. Frequency of intracranial hemorrhage and associated risk factors. Chest. 1997;111(5):1241-1245. doi:10.1378/chest.111.5.1241

10. Levine MN, Goldhaber SZ, Califf RM, et al. Hemorrhagic complications of thrombolytic therapy in the treatment of myocardial infarction and venous thromboembolism. Chest. 1995;108(4 Suppl):291S-301S.

11. Mikkola KM, Patel SR, Goldhaber SZ, et al. Increasing age is a major risk factor for hemorrhagic complications after pulmonary embolism thrombolysis. Am Heart J. 1997;134(1):69-72. doi: http://dx.doi.org/10.1016/S00028703(97)70108-X.

12. Sharifi M, Bay C, Mehdipour M. Moderate pulmonary embolism treated with thrombolysis (from the "MOPETT" Trial). Am J Cardiol. 2013;111(2):273-7. doi: 10.1016/j.amjcard.2012.09.027.

13. Wang C, Zhai Z, Shen YH, et al. Efficacy and safety of low dose recombinant tissue-type plasminogen activator for the treatment of acute pulmonary thromboembolism: a randomized, multicenter, controlled trial. Chest. 2010;137(2):254-62. doi: 10.1378/chest.09-0765.

14. Engelberger RP, Kucher N, et al. Ultrasound-assisted thrombolysis for acute pulmonary embolism: a systematic review. Eur Heart J. 2014;35:758-764. doi: http://dx.doi. org/10.1093/eurheartj/ehu029.

15. Kucher N, Boekstegers P, Baumgartner I, et al. Randomized, controlled trial of ultrasound-assisted catheter-directed thrombolysis for acute intermediate-risk pulmonary embolism. Circ. 2014;129(4):479-86. doi: 10.1161/ CIRCULATIONAHA.113.005544. 